\section{FRI0277 METFORMIN REDUCES SALIVARY GLAND INFLAMMATION BY CONTROLLING B CELL DIFFERENTIATION AND REGULATING BALANCE OF TH17 AND TREG CELL IN NON-OBESE DIABETIC MICE}

J.-W. Kim ${ }^{1}$, S.-M. Kim², J. Lee ${ }^{1}$, S.-K. Kwok ${ }^{1}$, J.H. Ju' ${ }^{1}$, S.-H. Park'. ${ }^{1}$ Division of Rheumatology, Department of Internal Medicine, School of Medicine, The Catholic University of Korea; ${ }^{2}$ Rheumatism Research Center, Catholic Research Institute of Medical Science, The Catholic University of Korea, Seoul, Korea, Republic Of

Background: Sjögren's syndrome (SS) is a systemic autoimmune disease that affects exocrine glands and lymphoid organs. B cell hyperactivity and imbalance between T helper 17 (Th17) cells and regulatory T (Treg) cells are involved in pathogenesis of SS. Metformin, a commonly used anti-diabetic drug, is found to have immunomodulatory effect via AMP-activated protein kinase enhanced inhibition of mTOR-STAT3 signalling.

Objectives: We examined the therapeutic effect of metformin on SS by using animal model of SS, non-obese diabetic (NOD) mice.

Methods: Metformin $(50 \mathrm{mg} / \mathrm{kg}$ ) or vehicle (saline) was given per oral every day from 11 weeks after birth until 20 weeks. Salivary flow rate (SFR) was addressed on every 2 or 3 weeks between 11 weeks and 20 weeks. Histologic analyses of salivary gland and spleen were performed on week 20. Expression of Inflammatory cytokine was determined by immunohistochemistry analysis and real-time PCR. Flow cytometry was performed with peripheral blood to examine Th17 and Treg cells and germinal centre (GC) B cell populations. Serum immunoglobulin level was measured by enzyme-linked immunosorbent assay. Splenic cells of NOD mice were treated with metformin or vehicle in vitro and cultured for 3 days. Results: SFRs of metformin-treated mice recovered, whereas SFRs of those treated with vehicle declined. Histologic examination of salivary gland showed decreased infiltration of lymphocytes and reduced expression of IL-6 and TNFalpha in metformin-treated mice. Relative expression of IL-6, TNF-alpha, and IL17 mRNA in salivary gland and spleen also declined in metformin-treated mice. Flow cytometric analysis revealed decreased Th1 and Th17 cells and increased Treg cells in peripheral blood of mice treated with metformin. In addition, GC B cells and immunoglobulin levels were reduced in peripheral blood of mice treated with metformin. Decreased Tfh cells and increased Tfr cells were observed from in vitro cultures of splenic cells treated with metformin

Conclusions: Metformin controls B cell differentiation and keeps balance between Th17 and Treg cells in NOD mice, in addition to reducing lymphocytic infiltration and inflammatory cytokine expression in salivary gland. Metformin has potential therapeutic effects on SS.

\section{REFERENCES:}

[1] Lee SY, Moon SJ, Kim EK, Seo HB, Yang EJ, Son HJ, et al. Metformin Suppresses Systemic Autoimmunity in Roquin ${ }^{\text {san/san }}$ Mice through Inhibiting B Cell Differentiation into Plasma Cells via Regulation of AMPK/mTOR/ STAT3. J Immunol 2017;198-2661-70.

[2] Pontarini E, Lucchesi D, Bombardieri M. Current views on the pathogenesis of Sjgren's syndrome. Curr Opin Rheumatol 2017.

Disclosure of Interest: None declared

DOI: 10.1136/annrheumdis-2018-eular.2815

\section{FRI0278 ALTERATIONS IN MEMORY AND VISIO-SPATIAL LEARNING IN A PRISTANE-INDUCED LUPUS BALB/C MICE}

J. Luciano Jaramillo, F. Sandoval Garcia, E.A. Martinez Garcia, M. Vazquez De Mercado, B.T. Martin Marquez. Universidad de Guadalajara, Guadalajara, Mexico

Background: Neuropsychiatric lupus is a condition that occurs in $30 \%-40 \%$ of systemic lupus erythematosus (SLE) patients and is associated with the presence of autoantibodies in central nervous system (CNS). The pristane-induced lupus in $\mathrm{BALB} / \mathrm{c}$ mice is an experimental model that presents clinical and immunological similarities to SLE pathogenesis and it can be proposed as model to analyse affectations in visio-spatial learning and memory.

Objectives: To evaluate short and long term visio-spatial learning and memory in Balb/c pristane-induced lupus using Barnes maze test.

Methods: Fourteen female Balb/c mice were studied: 4 controls (single intraperitoneal injection of $0.5 \mathrm{~mL} \mathrm{NaCl} 0.9 \%$ ), 5 treated with pristane (single intraperitoneal injection of $0.5 \mathrm{~mL}$ pristane) and 5 treated with pristane plus lipopolysaccharide (LPS) $3 \mathrm{mg} / \mathrm{kg} 4$ months post-administration of pristane. Short and long term visio-spatial learning and memory was evaluated in all groups at 7 weeks after the administration of LPS using Barnes maze test. The test consists of 3 phases: adaptation phase, acquisition phase and test phase. The short-term memory was evaluated during the acquisition phase (4 tests per day, D1-D4) and the long-term memory was evaluated 48 hour after the last test of the acquisition phase. Primary and total errors, primary and total latencies were analysed. The results are shown as a mean $\pm S D$. Kruscal-Wallis and Mann-Whitney $U$ test was used for statistical analysis.

Results: All mice treated with pristane showed higher latencies to find the target hole in Barnes maze compared with controls during the short-term memory test (STP, D1-D4). In the long-term memory test (LTP) the highest latency in seconds (s) was observed in the pristane plus LPS group $(64.6 \pm 59.1 \mathrm{~s})$ followed by the pristane group ( $62.4 \pm 69.6 \mathrm{~s}$ ) in comparation control group ( $7 \pm 3.6 \mathrm{~s})$.
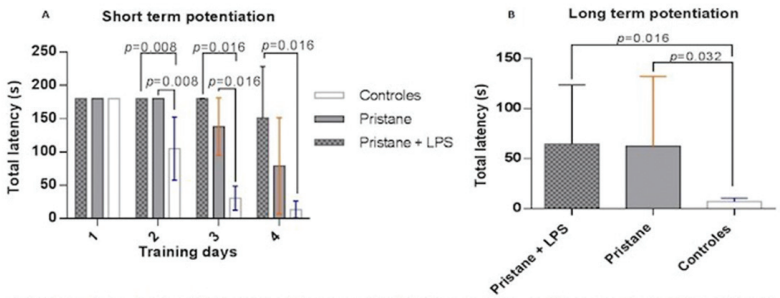

Abstract FRI0278 - Figure 1. Total. Latency in Barnes maze test. Total latency in seconds between mice groups (pristane, pristane + LPS and control) in STP (A) and LTP tests (B) in Barnes's maze

Conclusions: The increased latencies in the groups pristane plus LPS and pris tane with respect to controls suggests that the autoantibodies produced in this model could be involved in alterations of the CNS, modifying the learning and memory processes. These results also suggest that pristane may have the potential to alter the permeability of the blood-brain barrier by itself. However, more experimental studies are needed to confirm the results obtained in this study.

Disclosure of Interest: None declared

DOI: 10.1136/annrheumdis-2018-eular.5810

\section{FRI0279 DECREASED SPHINGOSIN-1-PHOSPHATE RECEPTOR 1 (S1P1) EXPRESSION IN ENDOTHELIAL PROGENITOR CELLS (EPCS) FROM SYSTEMIC LUPUS ERYTHEMATOSUS (SLE) PATIENTS}

J. Romo-Tena ${ }^{1}$, D. Gómez-Martín ${ }^{1}$, L.P. Blanco ${ }^{2}$, C. Carmona-Rivera² , R. CarazoVargas $^{1}$, J. Alcocer-Varela ${ }^{1}$, M.J. Kaplan². ${ }^{1}$ Immunology and Rheumatology, Instituto Nacional de Ciencias Médicas y Nutrición "Salvador Zubirán", Mexico City, Mexico; ${ }^{2}$ Systemic Autoimmunity Branch, National Institute of Arthritis and Musculoskeletal and Skin Diseases, National Institutes of Health, Bethesda, MD, USA

Background: SLE patients display an elevated cardiovascular risk when com pared to the general population. ${ }^{1}$ Interferon-alpha (IFN- $\alpha$ ) appears to drive atherogenesis in SLE at least in part, by promoting abnormal phenotype and function of EPCs. ${ }^{1}$ S1P1 is considered crucial for vasculogenesis and has been shown to attenuate IFN- $\alpha$ autoamplification by promoting IFN receptor 1 (IFNAR1) degradation. ${ }^{2,3}$

Objectives: To characterise S1P1 expression in EPCs from SLE patients and its putative association to type I IFNs effects on vasculogenesis.

Methods: SLE subjects that fulfilled SLE ACR classification criteria were recruited. Healthy controls were selected based on age and gender. Disease activity was assessed by SLEDAI-2K index. EPCs were quantified by flow cytometry in PBMC subsets as those cells that coexpress $C D 133^{+} / \mathrm{CD}_{3} 4^{+}$in the $\mathrm{CD} 3^{-}$ CD56 $/$ CD79b- gate. ${ }^{1}$ S1P1 expression was quantified in that subset. Contro PBMCs were cultured in endothelial cell (EC)-enrichment medium for 2 weeks Differentiated ECs were characterised by immunofluorescence (IF) as those cells that coexpress UEA-1 lectin and take up acetylated LDL. ${ }^{1}$ These cells were stimu lated with recombinant IFN- $\alpha$ in the presence or absence of the S1P1 agonist CYM-5442 and the S1P1 antagonist W-146 to assess the effect of this pathway on EC differentiation and type I IFN induced gene expression.3

Results: SLE and matched healthy controls ( $n=36 /$ group) were compared. Demographic and clinical characteristics are depicted in table 1

Abstract FRI0279 - Table 1. Demographic and Clinical features.

\begin{tabular}{lc}
\hline & Mean \pm SEM \\
\hline Age (years) & $32.2 \pm 1.35$ \\
Female (\%) & $34(94.44)$ \\
Time since SLE diagnosis (months) & $103.36 \pm 13.46$ \\
Time since remission (months) & $16.6 \pm 3.07$ \\
SLEDAl-2K (points) & $9.41 \pm 1.15$ \\
\hline
\end{tabular}

SLE patients displayed decreased absolute EPC numbers when compared to controls $(2463.43 \pm 450.07$ vs $39457.65 \pm 9063.34 \mathrm{EPCs} / \mathrm{ml}, \mathrm{p}<0.001)$. Decreased EPC numbers negatively correlated with SLEDAI-2K ( $r=-0.405, p=0.026)$. S1P1 expression was decreased in lupus EPCs when compared to controls $(48.27 \pm 1.55$ vs $60.89 \% \pm 1.66 \%$, 